\title{
Determination of Absolute Interferometric Phase using the Beam-Amplitude Ratio Technique
}

\author{
D. L. Bickel and W. H. Hensley \\ Sandia National Laboratories \\ Albuquerque, NM 87185-0537 USA \\ RECEIVED \\ MAR 151996 \\ Telephone: 505-845-8112, Facsimile: 505-845-9888, Email: dlbicke@sandia.gov, whhentogandia.gov
}

\begin{abstract}
Determination of the absolute phase difference (i.e., not modulo $2 \pi$ ) is a key problem in interferometric synthetic aperture radar (IFSAR) for topographic mapping. One way of solving this problem requires use of a technique different from the basic interferometry to resolve a "coarse" angle measurement that lies within the IFSAR ambiguity angle. The method investigated in this paper involves taking advantage of the difference in the amplitude ratio versus elevation angle that occurs when the elevation beams of the two IFSAR antennas are pointed in slightly different directions. The performance of the technique is a function of the angular separation of the two beams, the elevation beamwidth, and the symmetry of the two beam-amplitude patterns. The performance required of the technique is set by the ambiguity angle of the interferometer. This paper presents an analysis of the beam-amplitude ratio technique and shows experimental results.
\end{abstract}

\section{INTRODUCTION}

There continues to be growing interest in the area of height mapping using interferometric synthetic aperture radar (IFSAR). The ultimate goal of IFSAR is to provide an automatic way of producing elevation models in a true ground coordinate system or a WGS-84 coordinate system. This is accomplished in IFSAR by estimating an elevation angle relative to an antenna baseline-coordinate system, and then transforming to the desired ground or WGS coordinates. The elevation angle estimate is derived from the measurement of the interferometric phase.

One of the problems that must be overcome is determining the absolute ambiguity of the interferometric phase. This ambiguity comes from the fact that practical IFSAR systems do not adequately Nyquist sample the antenna elevation beamwidth. The consequence is that the elevation angle determined from the interferometric phase is unknown to some multiple of the spatial Nyquist frequency.

Two techniques have been proposed to address this issue in [1], and an overview of techniques is found in [2]. These methods can be thought of in two general categories. The first is to acquire additional Nyquist samples, such as multiple frequencies or baselines [3]. The second is to use non-phase information to aid the process. This could be tiepoints, or it could be a range-stereo technique as proposed in $[1,2]$.

In this paper we describe another technique that falls into the second category. This technique proposes applying a different elevation squint to each of the IFSAR antennas, as with amplitude monopulse, and using the relative intensity information to unambiguously determine the antenna pointing. Tying the antenna pointing to the IFSAR angle allows us to determine the absolute ambiguity in the IFSAR elevation angle measurement.

In the following sections we will briefly discuss the theory of operation and calibration. Finally, a couple of examples are presented.

\section{THEORY}

Principle of Operation

Figure 1 illustrates the fundamental operating principle of the beam-amplitude ratio technique. The idea is that the elevation angle to a target within the scene can be determined from ratio of the intensity between the two antennas. The angle measurement is not as sensitive as the angle determined from the interferometric phase. However, it only needs to be sensitive enough to determine the ambiguity.

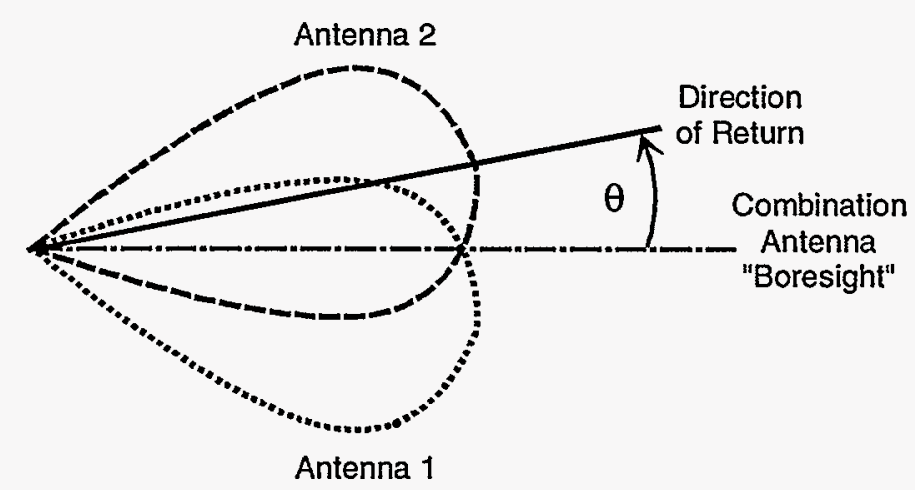

(a) Polar Plot of Antenna Beam Patterns

Figure 1: Principle of Beam-Ratio Technique 


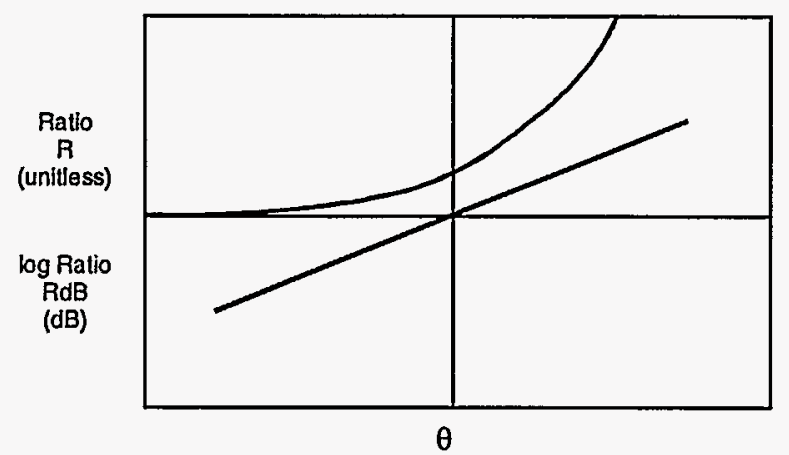

(b) Ratio of Antenna Beam Amplitudes vs. Elevation Angle $\theta$ Figure 1 (continued): Principle of Beam-Ratio Technique

As Figure 1 (a) shows, the peaks of the amplitude patterns of the two interferometer antennas are purposely mis-aligned in elevation. Figure 1 (b) shows that the ratio of the amplitude of a signal in channel 2 to its amplitude in channel 1 gives a measure of the angle of arrival of the signal. Also note that the log of the ratio is approximately linear.

Ignoring noise and assuming a Gaussian antenna pattern, we can follow the derivation of [4] and arrive at the following equation for the angle estimate:

$$
\theta=\frac{R_{d B}(\theta)-\left(P_{2, d B}-P_{1, d B}\right)}{\eta}
$$

where:

$\theta$ - is the estimate of the elevation angle

$R_{d B}(\theta)$ - is the beam ratio in $\mathrm{dB}$

$P_{2, d B}$ - is the 2-way peak power of antenna \#2

$P_{\mathrm{l}, d B}$ - is the 2-way peak power of antenna \#1

$\eta-$ is a scale factor

The scale factor, $\eta$, can be shown in this case to be:

$$
\eta=-12.04 \cdot\left(\frac{\theta_{0}}{\theta_{B}^{2}}\right)
$$

where:

$\theta_{0}$ - is angular separation between the two beams

$\theta_{B}$ - is half of the beamwidth of the one-way antenna pattern

\section{Engineering Tradeoffs}

Equation (2) indicates that it is desirable to separate the antenna beam pointing as far as possible. It is intuitive that there is a noise penalty paid by both the beam-ratio technique and the IFSAR due to the antenna separation that is not borne out in equation (2). The signal-to-noise (SNR) penalty that the beam-amplitude ratio technique has on the IFSAR height noise can be calculated using equation (22) from [5] and equations (25) and (31) from [6]. Figure 2 shows the IFSAR height noise increase due to the antenna separation for an example system configuration. The calculations in figure 2 are based on a system with a $15-\mathrm{dB}$ SNR at the beam center, a pair of antennas with 15-degree elevation beamwidths, a 1700-m ground swath at a 45degree incidence angle, and a short range $(5 \mathrm{~km})$. Note that at 3 degrees of beam separation there is only a $5 \%$ height noise penalty. This corresponds to an increase of only 0.4 $\mathrm{dB}$ in the system noise floor, which is acceptable for many system applications. The 3 degree beam separation gives a separation ratio of $1 / 5$ of the beamwidth. An offset of around $1 / 4$ to $1 / 5$ the beamwidth provides a good compromise between ratio sensitivity and system height noise.

The fact that the antennas are not aligned also leads to a reduction in the 3-dB beamwidth measured from the full two-antenna SNR. This is an important consideration when optimizing the design of an interferometer. Figure 3 shows the reduction of system beamwidth with beam separation for the case described above. Again, the penalty is small for a separation ratio of $1 / 5$, and quickly becomes unacceptable as the separation approaches $1 / 2$.

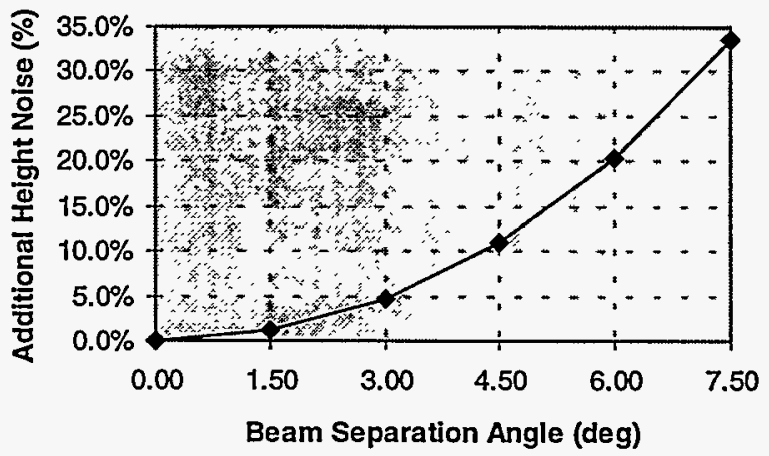

Figure 2: Additional Height Noise due to SNR Reduction from Beam Separation 


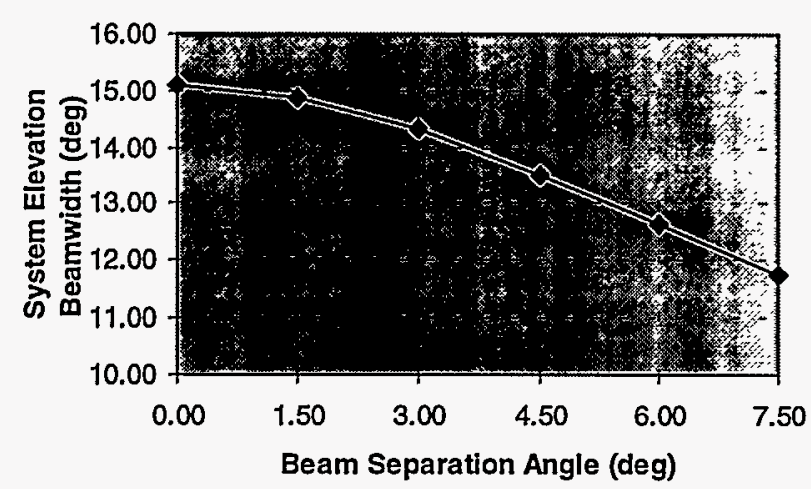

Figure 3: Reduction of System Beamwidth from Beam Separation

Performance Analysis

Increasing the beam separation angle also increases the noise for "coarse" angle estimate from the beam-ratio. Simulations were run for the case of a Gaussian antenna pattern with a $3^{\circ}$ beam separation and a $15^{\circ}$ elevation beamwidth. Figure 3 shows the effect of SNR and the effect of number of looks on the error in the estimate of the beam ratio, $R_{d B}(\theta)$. Figure 4 shows the effect that the antenna beam separation angle has on the error in the beam ratio.

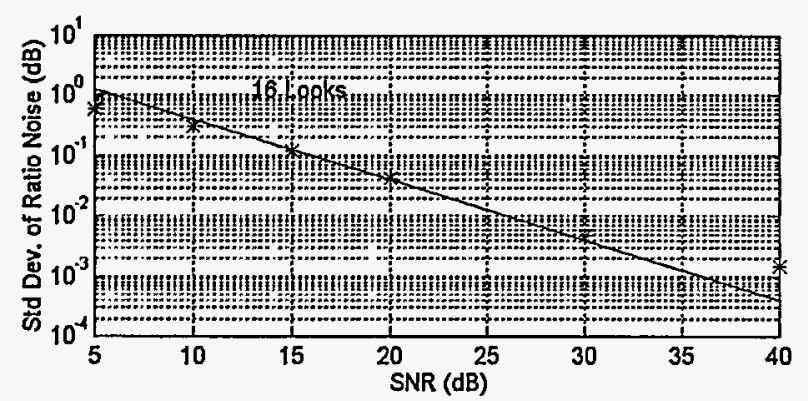

(a)

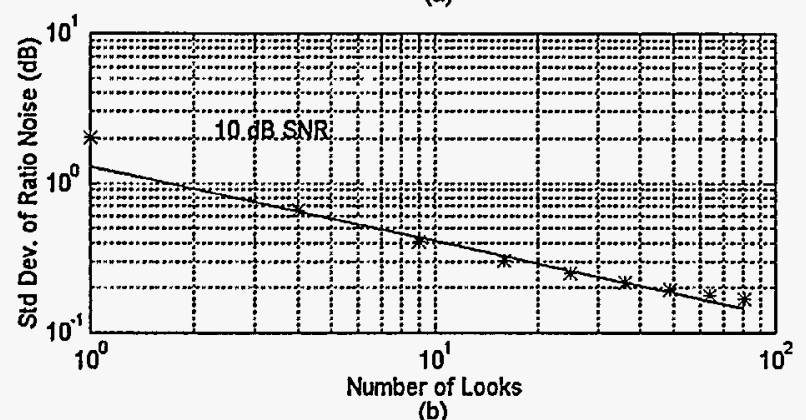

(b)

Figure 3: Effect of SNR and Number of Looks on Beam Ratio

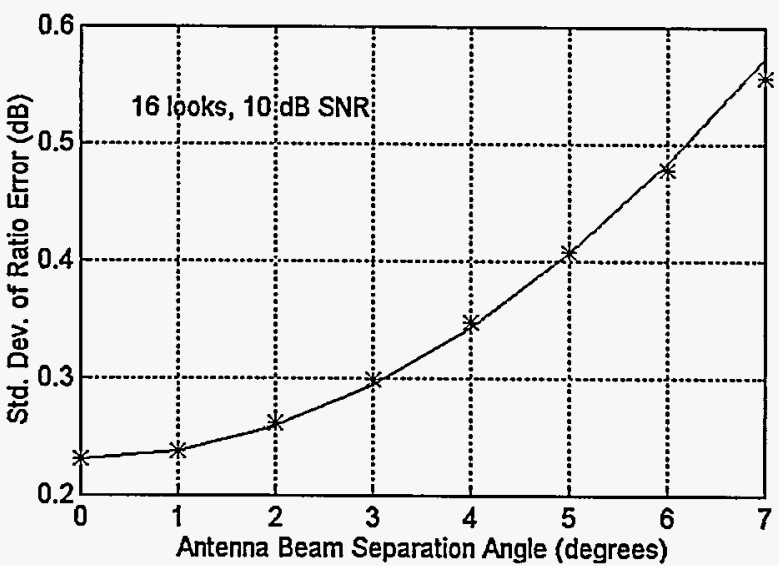

Figure 4: Effect of Beam Separation on Beam Ratio

The error in the angle estimate is found by dividing by the scale factor, $\eta$. The system design is based upon keeping the standard deviation of the angle error well below half of the IFSAR ambiguity angle. This is accomplished by a trade-off of number of looks, sufficient beam separation and height noise.

\section{CALIBRATION}

In order to determine the absolute ambiguity angle, we must be able to sufficiently calibrate the relative power between the two channels. Calibration error introduces an error into the term $\left(P_{2, d B}-P_{1, d B}\right)$ in equation (1). The accuracy with which we can calibrate the relative channel power influences how we set the scale factor; hence, ultimately how we set the antenna separation. This is a potentially more important influence on the choice of antenna separation than is the SNR consideration because we can always increase the number of looks in the SNR case.

\section{EXAMPLES}

In this section we give a couple of examples of applications of the beam-amplitude ratio technique. The first is an example from the Four Hills area southeast of Albuquerque, New Mexico. The second example is of the Washington Monument in Washington D. C. 
Absolute Phase Determination

The Four Hills formation is a small range of mountains with terrain height changes sufficient to require an absolutephase technique. For this example, the beam ratio was calculated for adjacent patches of the mountain terrain at a point where the number of ambiguities should have changed. Figure 5 shows that the third patch (aperture 26) has an ambiguity relative to the second patch (aperture 25) at the center of the scene (range bin number 1024). In Figure 6, the beam ratios for each patch are plotted versus the estimated angles from the respective unwrapped phases.

The patch with the ambiguity is shifted by an integer multiple of the ambiguity angle. From this figure we can see that the best fit is when the second patch is shifted by one ambiguity with respect to the first patch.

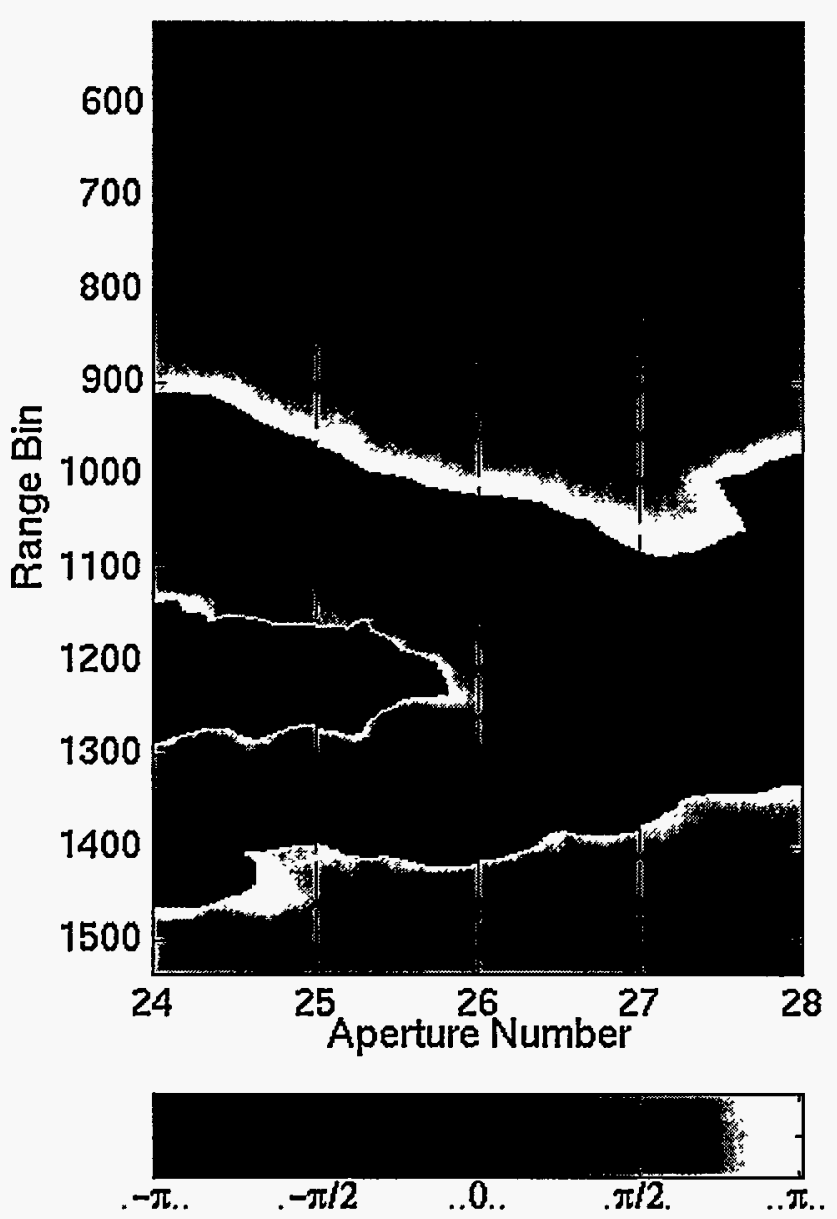

Figure 5: Phase Image showing $2 \pi$ Ambiguity Change at Center Range Bin

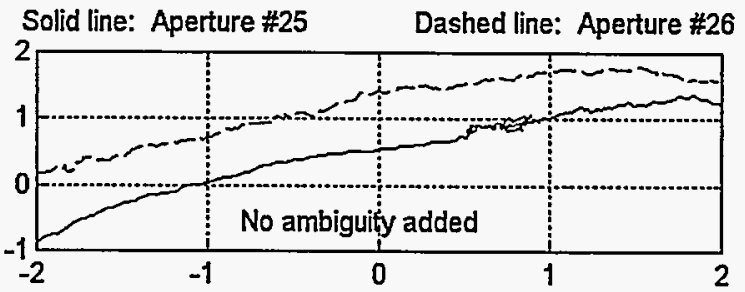

(a)

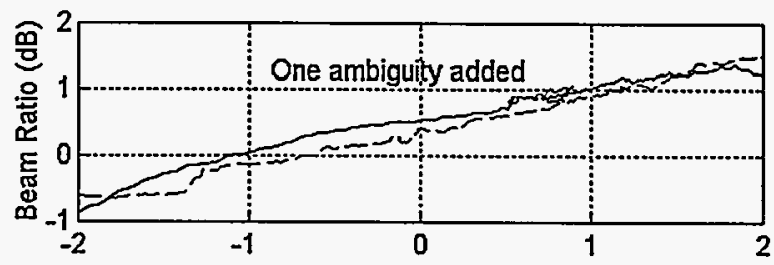

(b)

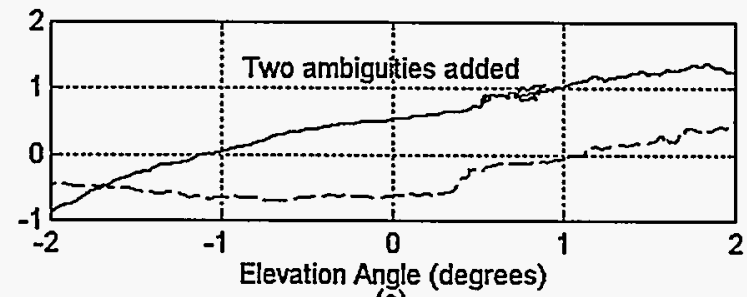

(c)

Figure 6: Example of beam-ratio for Mountain Terrain

\section{Detection of Phase Unwrapping Error}

The Washington Monument is an example of an entirely different application of the beam-ratio -- identifying a specific area within a scene that cannot be unwrapped using traditional path-following techniques. The beam-ratio technique can also be used for this more difficult and general problem if SNR and calibration issues are adequately addressed. Figure 7 illustrates this use of the technique.

Figure 7 (a) shows the height of the top of the Washington Monument estimated directly from the unwrapped phase difference. Because the top of the monument is less than $\pi$ radians below the surrounding terrain in the phase image, there is no indication that a wrap has occurred. Figure 7 (b) shows the beam-ratio computed for the same scene, scaled as a number of $2-\pi$ ambiguities. In the Monument area, the ratio value shows that 1.75 ambiguities must be added back. The indicated value must be rounded, giving an actual phase to be added of $2 \cdot 2 \pi$. Figure 7 (c) shows the estimated Monument height after the $4 \pi$ phase ambiguity has been added. The measured value is about 520 feet, while the actual height is $\mathbf{5 5 5}$ feet from the base to the top. 


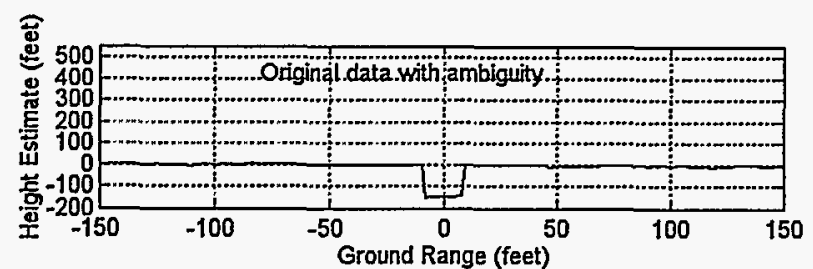

(a)

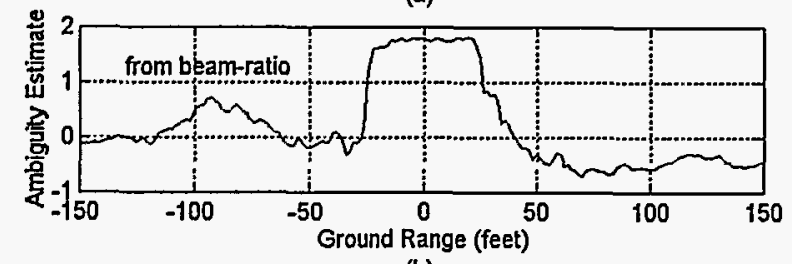

(b)

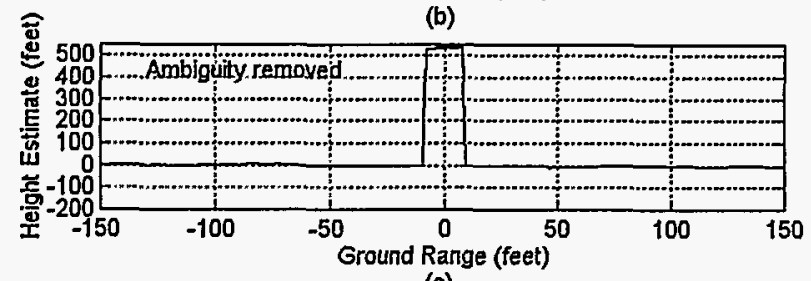

(c)

Figure 7: Use of the Beam-Ratio for Detecting Phase Unwrapping Error

\section{CONCLUSIONS}

The theory and examples show that the beam-amplitude ratio technique can be used as a method for determining the absolute ambiguity angle; hence, the absolute phase of the IFSAR system. The overall height noise penalty paid can be kept small. The key to successful ambiguity determination is the ability to measure and control the relative calibration between the two channels of the IFSAR system. The technique does not require the additional image formation processing of the multi-frequency approach or the extra hardware of the multi-baseline approach, and it avoids the computation-intensive correlation required for using range stereo.

In the case of a well-calibrated system with high SNR, the beam-ratio can also be used either to eliminate or seed the phase-unwrapping process. This will lead to much more robust system performance in areas with rapidly changing terrain.

\section{ACKNOWLEDGMENTS}

The beam-ratio technique is being developed for the Airborne Multi-sensor Pod System (AMPS) SAR for the Department of Energy. The authors wish to thank both the AMPS management and design staff for their assistance.

The examples in this paper were produced using an experimental capability on Sandia Laboratories' Twin Otter IFSAR. Thanks go to the flight test crew and the system developers for their assistance, and for answering all those questions!

This work was supported by the United States Department of Energy under Contract DE-AC0494AL85000.

\section{REFERENCES}

[1] S. N. Madsen, "On absolute phase determination techniques in SAR interferometry", Proceedings of the SPIE, Orlando, Florida., 19-21 April, 1995, Vol. 2487, pp. 393-401.

[2] W. G. Carrara, R. S. Goodman, R. M. Majewski, section 9.3.5 of Spotlight Synthetic Aperture Radar, Artech House, 1995.

[3] C. V. Jakowatz, D. E. Wahl, and P. A. Thompson, "Ambiguity Resolution in SAR Interferometry by Use of Three Phase Centers", Proceedings of S.P.I.E Aerosense Conference, 8-12 April, 1996, Orlando, Florida.

[4] L. G. Bullock, G. R. Oeh, and J. J. Sparagna, "An Analysis of Wide-Band Microwave Monopulse DirectionFinding Techniques", IEEE Trans. on Aerospace and Electronic Systems, Vol. AES-7, No. 1, Jan., 1977, pp. 188-202.

[5] D. Just and R. Bamler, "Phase statistics of interferograms with applications to synthetic aperture radar", Applied Optics, Vol 33, No. 20, July 10, 1994, pp. 4361-4368.

[6] E. Rodriguez and J. M. Martin, "Theory and design of interferometric synthetic aperture radars", IEE Proceedings- $F$, Vol. 139, No. 2, April, 1992, pp. 147-159.

\section{DISCLAIMER}

This report was prepared as an account of work sponsored by an agency of the United States Government. Neither the United States Government nor any agency thereof, nor any of their employees, makes any warranty, express or implied, or assumes any legal liability or responsibility for the accuracy, completeness, or usefulness of any information, apparatus, product, or process disclosed, or represents that its use would not infringe privately owned rights. Reference herein to any specific commercial product, process, or service by trade name, trademark, ence herein to any specific does not necessarily constitute or imply its endorsement, recommendation, or favoring by the United States Government or any agency thereof. The views mendation, or favoring by the United States Government or any agency thereof. 